\title{
Morrinhosuchus luziae, um novo Crocodylomorpha Notosuchia da Bacia Bauru, Brasil
}

\author{
Fabiano Vidoi Iori' ${ }^{1}$ \& Ismar de Souza Carvalho ${ }^{1}$
}

\begin{abstract}
Resumo Uma nova espécie de Crocodylomorpha Notosuchia proveniente da Bacia Bauru, Formação Adamantina (Cretáceo Superior, Turoniano-Santoniano), é descrita neste estudo. O fóssil consiste das porções distais do crânio e da mandíbula. Observam-se dentes posteriores de coroas globosas e de seção transversal circular. Dentre os notossúquios descobertos na Bacia Bauru apenas Mariliasuchus apresenta dentes similares, no entanto, a distribuição destes dentes entre outras autapomorfias sustentam a definição do novo táxon. Esta descoberta amplia o número e a diversidade de formas dos Notosuchia e corroboram o sucesso na distribuição deste clado pelo Gondwana e sua ocupação em vários nichos ecológicos durante o Cretáceo.
\end{abstract}

Palavras-chave: Crocodylomorpha, Notosuchia, Morrinhosuchus luziae, Formação Adamantina, Bacia Bauru

\begin{abstract}
Morrinhosuchus Luziae, a new Crocodylomorpha Notosuchia from the Bauru Basin, Brazil. A new species of a Crocodylomorpha Notosuchia from the Bauru Basin, Adamantina Formation (Upper Cretaceous, Turonian-Santonian), Brazil is described. The fossil consists of the distal portions of skull and mandible. The posterior teeth present globoid crown and circular cross-section. Among the notosuchians discovered in the Bauru Basin, only Mariliasuchus presents similar teeth, however, the arrangement of these teeth, besides other autapomorphies, allow the establishment of a new taxa. This fossil widens the number and diversity of Notosuchia, showing the success of this clade throughout Gondwana and several ecological niches during the Cretaceous.
\end{abstract}

Keywords: Crocodylomorpha, Notosuchia, Morrinhosuchus luziae, Adamantina Formation, Bauru Basin.

INTRODUÇÃO Os notossúquios foram crocodiliformes cretácicos, com uma ampla distribuição e diversidade no contexto gondwânico. Ocuparam vários nichos ecológicos, com formas onívoras e algumas possivelmente herbívoras. Apresentam características peculiares, como crânio alto e lateralmente achatado, número de dentes reduzidos e órbitas dispostas lateralmente. Observa-se também uma grande variação morfológica entre as espécies deste grupo, principalmente no rostro, e nos padrões de dentição que incluem heterodontia, e alta especialização dentária (Carvalho \& Bertini 1999).

$\mathrm{Na}$ Bacia Bauru, de onde provém o espécime em estudo, foram descritas formalmente seis espécies de notossúquios: Sphagesaurus huenei Price 1950; Mariliasuchus amarali Carvalho \& Bertini 1999; Adamantinasuchus navae Nobre \& Carvalho 2006; Mariliasuchus robustus Nobre, Carvalho, Vasconcellos \& Nava 2007; Sphagesaurus montealtensis Andrade \& Bertini 2008 e Armadillosuchus arrudai Marinho \& Carvalho 2009. De acordo com os estudos de Sereno et al. (2003), Pol (2003), Zaher et al. (2006), Andrade \& Bertini (2008), as análises filogenéticas indicam que o gênero Baurusuchus também poderia ser incluído em Notosuchia.

O presente estudo analisa uma nova espécie de um Crocodylomorpha Notosuchia, representada por apenas um espécime, descoberto no município de Monte Alto, Estado de São Paulo, Brasil. Embora o fóssil con- sista apenas das regiões distais do crânio e da mandíbula, foi possível o levantamento de vários dados de relevância taxonômica que possibilitaram a definição da nova forma.

GEOLOGIA A Bacia Bauru localiza-se no centro sul da Plataforma Sul-Americana (Fig. 1) e seu preenchimento ocorreu em condições climáticas semi-áridas a áridas, entre o Coniaciano e o Maastrichtiano (Fernandes \& Coimbra 2000). Dias-Brito et al. (2001), baseados em minuciosos estudos micropaleontológicos, sugerem dois intervalos de sedimentação para parte da sequência (Turoniano-Santoniano e Maastrichtiano), que representa um pacote continental avermelhado dominado por arenitos, siltitos, argilitos/folhelhos, exibindo calcretização em certos níveis, depositados em diversos contextos ambientais, tais como eólico, aluvial, fluvial e lacustre (Dias-Brito et al. 2001, Fernandes \& Coimbra 1996, Fernandes \& Coimbra 1994, Batezelli et al. 2003, Batezelli et al. 2005).

Na região do município de Monte Alto, de onde provém o fóssil em análise, ocorrem duas unidades litoestratigráficas distintas: as formações Adamantina e Marília (Fig. 2). Ambas são compostas por litótipos essencialmente clásticos. A nova espécie analisada neste estudo provém de um afloramento da Formação Adamantina, localizado próximo ao Morrinho de Santa Luzia, na área rural do município. O afloramento con- 


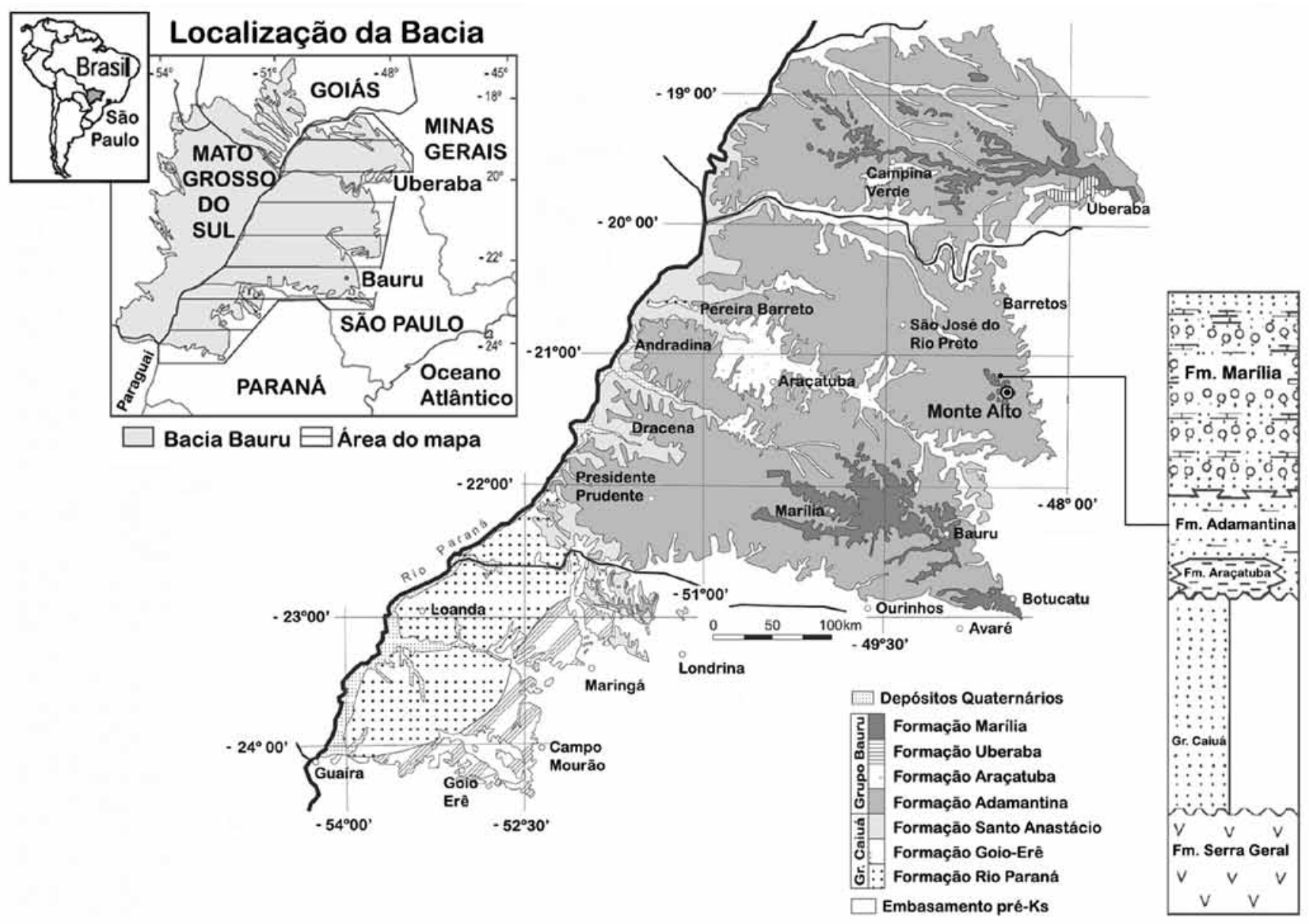

Figura 1 - Mapa geológico da parte oriental da Bacia Bauru (modificado de Fernandes \& Coimbra 2000) e coluna litoestratigráfica do Grupo Bauru no estado de São Paulo e seu contato com o Grupo Caiuá (Batezelli et al. 2003).

siste em arenitos avermelhados, sem estruturas internas observadas, ocorrência de nódulos e concreções carbonáticas em vários níveis, e localmente, camadas com evidências de intensa bioturbação.

MATERIAS E MÉTODOS O espécime em estudo foi encontrado em uma rocha já em processo de intemperização, com parte do ramo mandibular esquerdo articulado com a parte distal do crânio. A porção preservada sofreu desarticulação durante a fase bioestratinômica da fossilização, no entanto a superfície ventral do crânio e o ramo mandibular esquerdo estão bem preservados e com suas estruturas definidas, permitindo o levantamento de dados confiáveis na definição deste novo táxon.

A classificação da nova espécie baseou-se em estudos comparativos com os de Rusconi (1933), Wu \& Sues (1996), Gomani (1997), Carvalho \& Bertini (1999), Buckley et al. (2000), Nobre \& Carvalho (2002), Martinelli (2003), Pol (2003), Sereno et al. (2003), Vasconcellos \& Carvalho (2005), Nobre \& Carvalho (2006), Zaher et al. (2006), Nobre et al. (2007), Andrade \& Bertini (2008) e Marinho \& Carvalho (2009).
A terminologia osteológica baseou-se em Iordansky (1973) e Carvalho et al. (2004).

\section{PALEONTOLOGIA SISTEMÁTICA E DESCRIÇÃO}

Crocodylomorpha Walker 1970

Crocodyliformes Hay 1930

Mesoeucrocodylia Whetstone \& Whybrow 1983

Morrinhosuchus gen. nov.

Etimologia O nome genérico Morrinhosuchus faz alusão ao local de coleta do fóssil, nas proximidades do Morrinho de Santa Luzia, uma feição geomorfológica, utilizado como mirante e sobre o qual se construiu uma capela em devoção à Santa Luzia.

Diagnose A mesma que para a espécie

Morrinhosuchus luziae gen. nov. et sp. nov.

Etimologia O designativo específico, luziae, se refere à Santa Luzia.

Holótipo MPMA 07-0009/01 (Museu de Paleontologia de Monte de Alto). Porções distais do crânio e da 


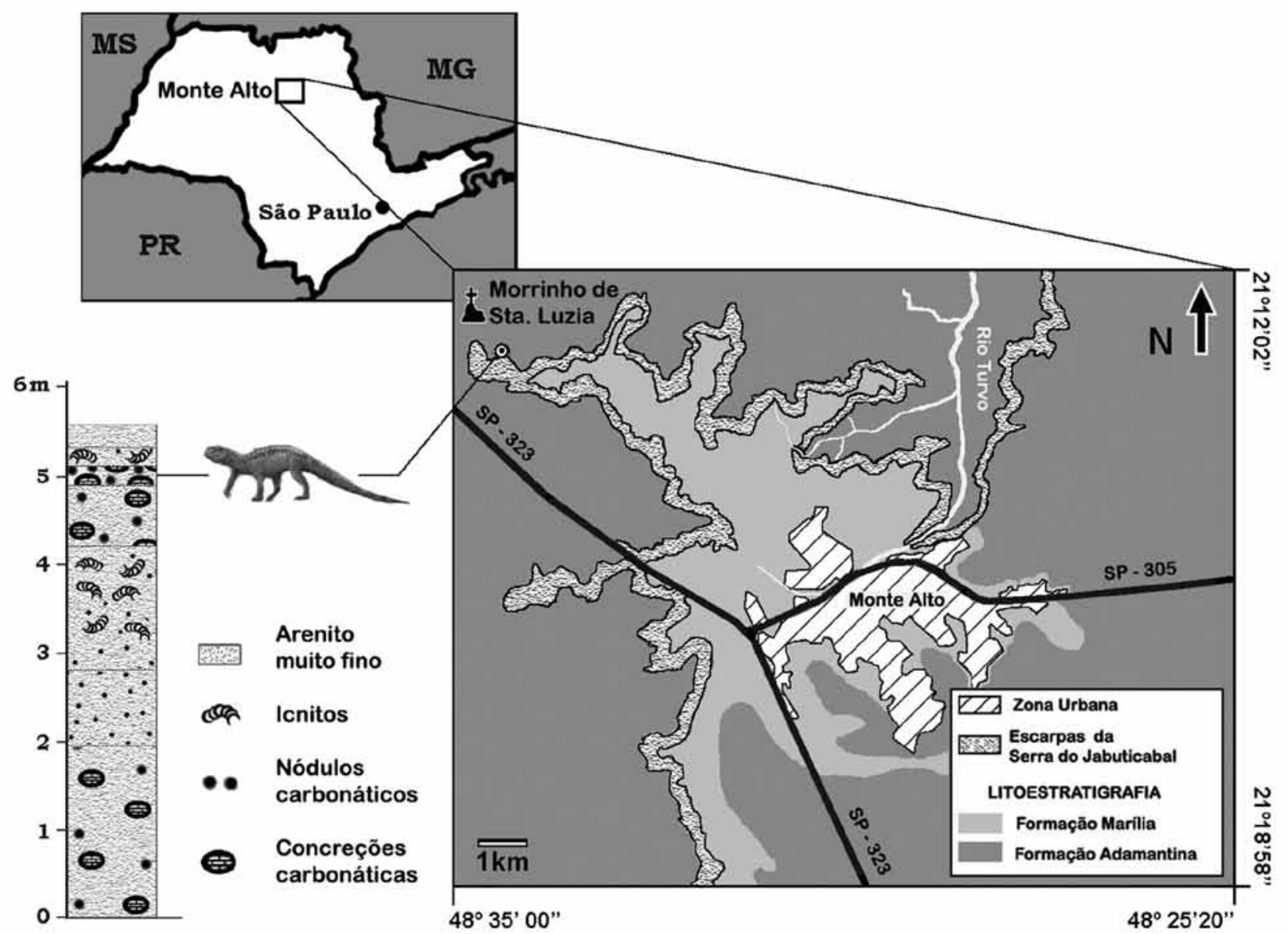

Figura 2 - Mapa geológico da região do município de Monte Alto (modificado de Iori 2008) e perfil estratigráfico do afloramento de coleta do fóssil MPMA 07-00009/01.

mandíbula (Figs. 3 e 4).

Localidade $13 \mathrm{~km}$ de Monte Alto, próximo ao sopé do Morrinho de Santa Luzia (21 $11^{\circ}$ ' 13,02" S e 48 34' 10,42 ”) na área rural do município de Monte Alto, Estado de São Paulo, Brasil.

Contexto estratigráfico Bacia Bauru, Formação Adamantina, Cretáceo Superior (Turoniano-Santoniano).

Diagnose Mandíbula arqueada antero-posteriormente e com superfície dorsal côncava, anteriormente estreita e projetada. A sínfise é longa, com pouca participação do esplenial, e os ramos mandibulares bastante divergentes. Cada ramo apresenta seis dentes posteriores (coroas globosas e de seção transversal circular) dispostos em uma linha reta, sendo os três últimos dentes os maiores. Dentário com uma pequena expansão lateral, formando uma crista que demarca os planos lateral e latero-ventral. Prémaxilar lateralmente intumescido participando amplamente da narina externa; apresenta pelo menos dois alvéolos circulares (um de $5 \mathrm{~mm}$ de diâmetro e outro com $2 \mathrm{~mm}$ de diâmetro). Maxilar lateralmente quase vertical e sem exposição na superfície dorsal. É longo e estreito com seis alvéolos circulares em sua superfície ventral. Nasais longos e estreitos; porção distal do palatino chato e estreito contactando latero-anteriormente o maxilar. Presença de fenestra antero-orbital e de um pequeno entalhe lateral na união da pré-maxila com a maxila.

\section{Morrinhosuchus gen. nov.}

Etymology The generic name, Morrinhosuchus is a reference to the sampling area, in the proximity of Morrinho de Santa Luzia, a hill where was built a chapel in glorification to the Saint Luzia.

Diagnosis The same as for species

\section{Morrinhosuchus luziae gen. nov. et sp. nov.}

Etymology The specific designative, luziae, is a reference to the Saint Luzia.

Holotype MPMA 07-0009/01 (Museu de Paleontologia de Monte de Alto). Distal portions of the skull and mandible (Figs. 3 and 4).

Locality $13 \mathrm{~km}$ from Monte Alto, near the foothill of 


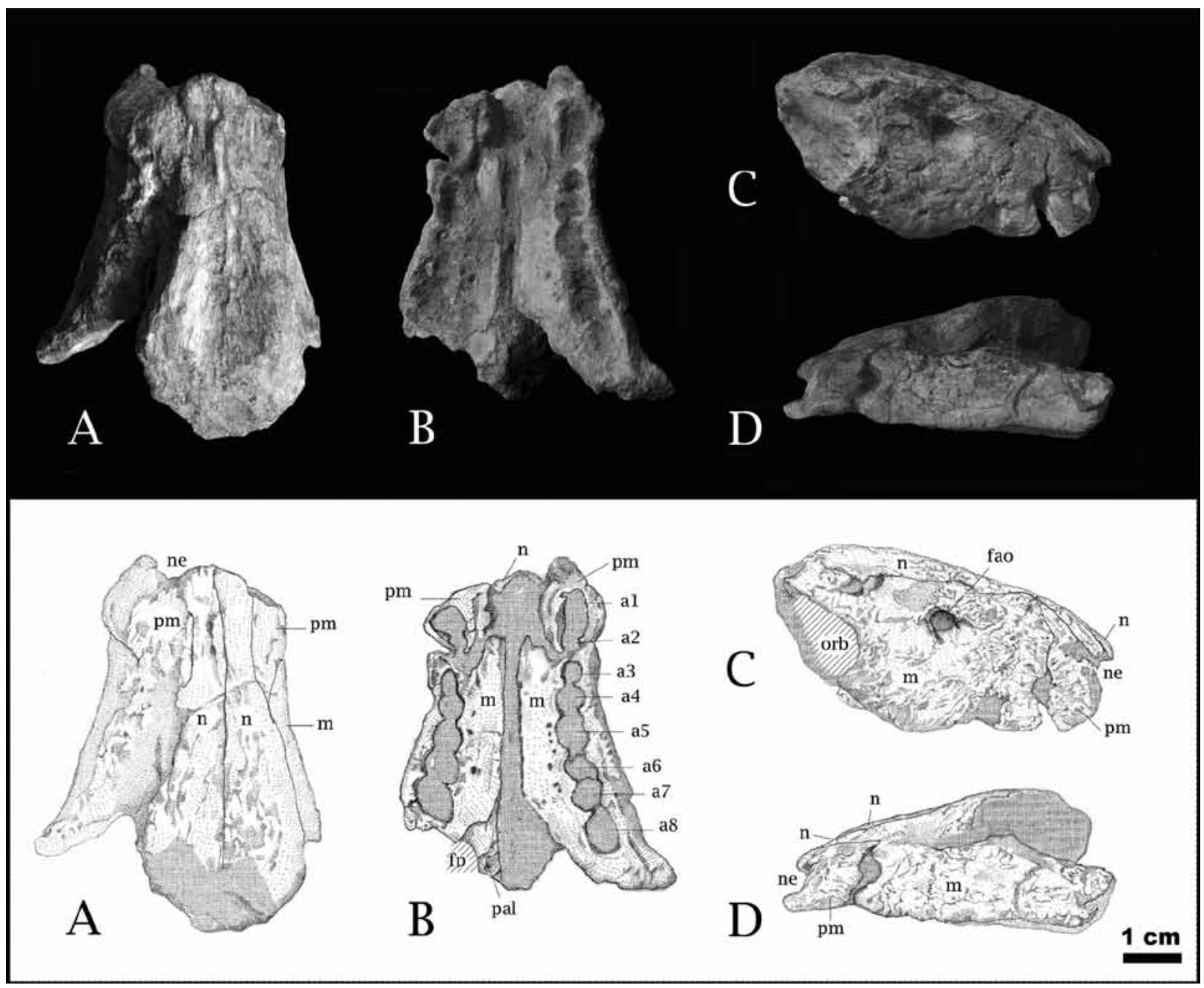

Figura 3 - Morrinhosuchus luziae gen. nov. et sp. nov. Fotografias e desenhos esquemáticos do crânio em vistas dorsal (A), ventral (B), lateral direita $(C)$ e lateral esquerda (D). Abreviações: a - alvéolo; fao fenestra antero-orbital; $\boldsymbol{f} \boldsymbol{p}$-fenestra palatal; $\boldsymbol{m}$ - maxilar; $\boldsymbol{n}$-nasal; $\boldsymbol{n e}$-narina externa; orb-órbita; pal-palatino; pm-pré-maxilar.

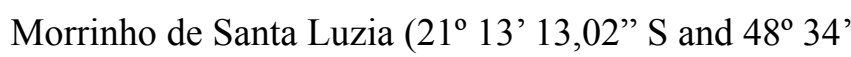
10,42 ”W), in the rural area of Monte Alto County, São Paulo State, Brazil.

Stratigraphic context Bauru Basin, Adamantina Formation, Upper Cretaceous (Turonian-Santonian).

Diagnosis Mandible antero-posteriorly arched and concave in dorsal surface, anteriorly is narrow and projected. The synfisis is lengthy and with discrete participation of the splenial. The mandibular branches are very divergent and each branch presents six posterior teeth (globoid crown and circular cross-section) disposed in a straight line; the last three teeth are bigger than the first ones. Dentary exhibits a little lateral expansion, forming an antero-posteriorly crest, which limits the lateral and latero-ventral planes. Premaxilla laterally expanded and bordering the major part of the external nostril. There are at least two circular alveolus (the first with $5 \mathrm{~mm}$ of diameter and the other with 2 $\mathrm{mm}$ of diameter). Maxilla is lengthy and narrow, later- ally is almost vertical and without exposition in the dorsal surface; presents six circular alveolus in the ventral surface. Nasals are long and narrow; the distal portion of palatine is flat and narrow and contacts the maxilla latero-anteriorly. There is an antorbital fenestra and a small lateral notch in the premaxilla-maxilla junction.

Descrição $O$ espécime aqui estudado apresenta apenas as porções distais do crânio e mandíbula. $\mathrm{O}$ crânio se limita à região anterior à órbita e a parte preservada da mandíbula não ultrapassa a margem anterior da fenestra mandibular. Em vista palatal observa-se que houve um deslocamento dorso-ventral e os ossos do palato direito não estão articulados aos ossos do palato esquerdo. Aparentemente o prémaxilar e o maxilar esquerdos sofreram um deslocamento lateral, causando uma ruptura nos ossos da superfície dorsal. Em vista ventral, os pré-maxilares parecem ter sofrido um leve deslocamento e estão desarticulados dos maxilares. 


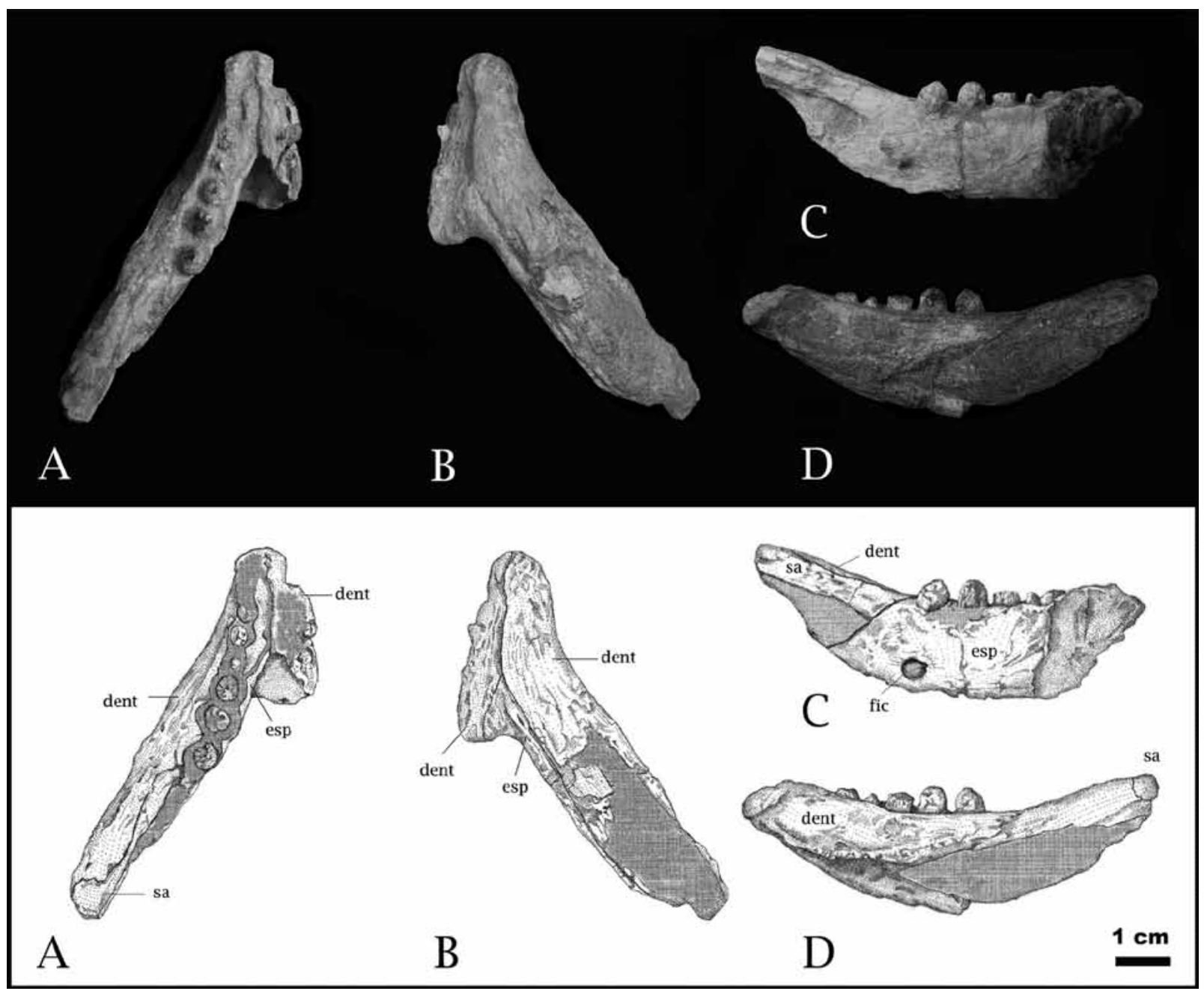

Figura 4 - Morrinhosuchus luziae gen. nov. et sp. nov. Fotografias e desenhos esquemáticos da mandíbula em vistas dorsal (A), ventral (B), lateral direita (C) e lateral esquerda (D). Abreviações: dent - dentário; esp-esplenial; fic-forâmen intramandibular caudal; sa-surangular.

PRÉ-MAXILAR Ambos não estão preservados integralmente. Em vista ventral observam-se pelo menos dois alvéolos, um pequeno, próximo ao contato com o maxilar, de aproximadamente $2 \mathrm{~mm}$ de diâmetro e anterior a ele estaria alojado um dente provavelmente caniniforme, justificando a intumescência observada lateralmente. Esta intumescência marca toda a extensão lateral do pré-maxilar, começando próximo à narina externa e estendendo-se até a sutura com o maxilar. A linha de sutura com o maxilar está marcada por um entalhe que se inicia na margem bucal e se estende dorsalmente além da metade do comprimento da sutura. Tal entalhe pode ter sido resultante do deslocamento dos maxilares, mas em ambos os lados estas estruturas se mostram muito simétricas.

MAXILAR Sua face lateral é praticamente vertical e perpendicular à superfície dorsal do rostro. Contacta o nasal dorsalmente e não está exposto na superfície dorsal; anteriormente contacta o pré-maxilar, onde ocorrem alguns sulcos bem rasos, ornamentando a região que antecede a fenestra antero-orbital. Há alguns foramens neuro-vasculares dispostos acima da borda bucal. A superfície ventral está mais bem preservada, é alongada antero-posteriormente e estreita latero-medialmente. $\mathrm{O}$ contato com o palatino é curto e posteromedial. Nenhum dente está preservado, havendo, entretanto seis alvéolos circulares dispostos quase paralelos à sutura entre os maxilares; os dois primeiros alvéolos são de menor diâmetro (aproximadamente $4 \mathrm{~mm}$ para o primeiro e $5 \mathrm{~mm}$ para o segundo), os demais apresentam diâmetros de aproximadamente $6 \mathrm{~mm}$. Possui um pequena reentrância próximo ao primeiro alvéolo. Pequenos foramens são observados adjacentes à margem interna dos alvéolos.

NASAL É tabular, longo e estreito, visto nas superfícies dorsal e lateral. Participa da margem superior da narina externa. A porção lateral contacta ventralmente o maxilar e o pré-maxilar. Seu contato com os frontais, lacrimal e demais ossos não está claro.

PALATINO Apenas a porção mais distal do palatino direito se preservou, a qual é achatada e estreita, contactando a maxila antero-lateralmente. A borda anterior 
da fenestra mandibular marca o final da sutura.

FENESTRA ANTERO-ORBITAL Tem o formato levemente ovalado e situa-se entre a sutura pré-maxilarmaxilar e a margem anterior da órbita. Sua margem anterior e quase toda extensão da margem ventral é composta pelo maxilar. Os limites dos ossos envolvidos no restante da borda não estão bem definidos.

A mandíbula teve preservada a maior parte do dentário e esplenial esquerdos e uma pequena parte do ramo mandibular direito. Em vista dorsal se mostra estreita anteriormente com uma sínfise longa, em que há a participação do espenial e sob forma de ramos divergentes. A porção distal se encaixa entre os caniniformes.

DENTÁRIO Apresenta uma pequena expansão lateral em sua porção anterior, formando uma pequena crista que se estende antero-posteriormente, demarcando os planos lateral e latero-ventral naquela região. Tal crista se torna quase imperceptível na altura do antepenúltimo dente.

Em vista lateral apresenta um arqueamento para cima logo após o último dente e a superfície dorsal é côncava. Participa da maior parte da sínfise.

A extremidade distal não está preservada, não sendo possível precisar a quantidade correta de dentes. Seis dentes estão preservados e dispostos em linha reta. Os dois últimos tiveram as coroas preservadas; são dentes rasos e globosos, com seção transversal circular. Não ultrapassam $4 \mathrm{~mm}$ de diâmetro e apresentam parte do esmalte preservado. $\mathrm{O}$ antepenúltimo dente apresenta a base da coroa e este teria o mesmo tamanho dos dentes posteriores. Os três dentes anteriores só possuem as raízes e são menores, com diâmetro de aproximadamente $2,5 \mathrm{~mm}$.

ESPLENIAL Margeia internamente os três últimos dentes da mandíbula, participa da sínfise mandibular.

FORÂMEN INTRAMANDIBULAR CAUDAL É bem pequeno e circular, localiza-se no dentário na altura do bordo posterior do último dente.

\section{COMPARACÕES E DISCUSSÕES}

Morrinhosuchus luziae gen. nov. et sp. nov. tem entre suas peculiaridades a presença de dentes globosos, os quais ocorrem em outros notossúquios da Bacia Bauru, tais como Mariliasuchus amarali e Mariliasuchus robustus (Carvalho \& Bertini 1999, Nobre et al. 2007). Mariliasuchus amarali possui uma pré-maxila bulbosa, caracterizada pela constrição lateral na altura dos dentes caniniformes; além desta, são características distintas para Morrinhosuchus luziae gen. nov. et sp. nov.: presença de fenestra maxilo-palatal; um palatino amplo e largo em sua porção distal; ausência de vestígio da fenestra antero-orbital; um dentário largo anteriormente com os dentes (exceto o $1^{\circ}$ dente) dispostos sobre uma linha parabólica (Carvalho \& Bertini 1999, Zaher et al. 2006). Mariliasuchus robustus se caracteriza pela robustez do crânio e divide com Morrinhosuchus luziae gen. nov. et sp. nov. uma mandíbula arqueada verticalmente, sendo que em M. robustus tal característica é mais marcante (Nobre et al. 2007).

Mariliasuchus apresenta dentes incisiformes, caniniformes e globosos. Carvalho \& Bertini (1999) apontam para $M$. amarali 3 dentes pré-maxilares (sendo o terceiro caniniforme) e 3 maxilares. Vasconcellos \& Carvalho (2005), com base em outros espécimes, atribuem para M. amarali um total de 9 dentes ( 3 pré-maxilares e 6 maxilares). Zaher et al. (2006) descrevem a mesma quantidade de dentes, no entanto, a distribuição difere, sendo 4 dentes pré-maxilares e 5 maxilares. Tal divergência ocorre no posicionamento do $1^{\circ}$ dente pós-caniniforme. $M a-$ riliasuchus robustus, segundo Nobre et al. (2007), também apresenta 9 dentes (3 pré-maxilares e 6 maxilares). Morrinhosuchus luziae gen. nov. et sp. nov. teve somente os seis dentes posteriores da mandíbula preservados, os quais possuem aspectos globosos. Os dentes maxilares e pré-maxilares não se preservaram, mas seus alvéolos são individualizados e permitiram a contabilização dos dentes (pelo menos 2 pré-maxilares e 6 maxilares). Ocorreriam 7 dentes pós-caniniformes em Morrinhosuchus luziae gen. nov. et sp. nov. (o último dente do pré-maxilar e os dentes maxilares). Já em ambas as espécies de Mariliasuchus ocorrem 6 dentes pós-caniniformes.

Sphagesaurus huenei Price 1950, Sphagesaurus montealtensis Andrade \& Bertini 2008 e Adamantinasuchus navae Nobre \& Carvalho 2006 também apresentam 7 dentes pós-caniniformes, mas estas espécies, assim como Armadillosuchus arrudai Marinho \& Carvalho 2009, possuem dentes de seção transversal ovalada e dispostos obliquamente ao eixo sagital do crânio. Já em Morrinhosuchus luziae gen. nov. et sp. nov. os contornos circulares dos alvéolos maxilares nos permite inferir que os seus respectivos dentes teriam aspectos similares aos da mandíbula.

Sphagesaurus huenei como Morrinhosuchus luziae gen. nov. et $\mathrm{sp}$. nov. têm o mesmo número de dentes superiores, sendo dois pré-maxilares (o primeiro hipertrofiado) e seis dentes no maxilar. Porém em $S$. huenei os dentes maxilares são de coroa triangular com estrias longitudinais na face antero-labial e uma carena denticulada na face postero-lingual. Quanto à mandíbula de $S$. huenei conhece-se apenas a parte mais distal do dentário, onde se preservaram os quatro primeiros dentes, sendo que os três primeiros são cônicos e aumentam de tamanho no sentido antero-posterior (Pol 2003). Dentes com quilhas denticuladas ocorrem também em Adamantinasuchus navae; este possui três carenas nos dentes do maxilar. A. navae possui três dentes no pré-maxilar, sendo os dois primeiros cônicos e o terceiro hipertrofiado; o maxilar apresenta sete dentes com tamanhos e formas diferenciadas e de aspectos molariformes (Nobre \& Carvalho 2006).

Sphagesaurus montealtensis, segundo Andrade \& Bertini (2008), apresenta apenas um dente caniniforme no pré-maxilar e sete dentes no maxilar de implantação oblíqua. Quase todos os dentes da mandíbula estão preservados, sendo que os cinco últimos dentes apresentam implantação oblíqua e presença de carena na superfície antero-labial, diferentemente de Morrinhosuchus luziae gen. nov. et sp. nov., cujos dentes são circulares e de coroas globosas. S. montealtensis 
apresenta uma fenestra antero-orbital pequena e circular semelhante à observada em Morrinhosuchus luziae gen. nov. et sp. nov., mas nesse último tal estrutura é relativamente mais afastada do início da órbita.

Candidodon itapecuruense Carvalho \& Campos 1988 possui heterodontia verdadeira, com dentes cônicos, espatulados, caniniformes e molariformes (uma cúspide central espatulada circundada por dentículos na base da coroa) (Carvalho 1993, Nobre \& Carvalho 2002). Os padrões dos dentes molariformes de $C$. itapecuruense são bastante semelhantes aos encontrados em Malawisuchus mwakasyungutiensis Gomani 1997. Dentes multicuspidados ocorrem também em Chimaerasuchus paradoxus Wu, Sues \& Sun 1995 (dentes molariformes com três fileiras longitudinais de sete cúspides recurvadas), Simosuchus clarki Buckley, Brochu, Krause \& Pol 2000, Uruguaysuchus aznarezi Rusconi 1933 e Uruguaysuchus terrai Rusconi 1933 (Wu \& Sues 1996, Buckley et al. 2000, Rusconi 1933). Estes dois últimos gêneros dividem padrões semelhantes na forma dos dentes multicuspidados (são dentes espatulados fortemente comprimidos lateralmente, com cúspides enfileiradas longitudinalmente). Ambas espécies de Uruguaysuchus apresentam uma série dentária bastante numerosa, U. aznarezi possui 13 dentes (4 no pré-maxilar e 9 no maxilar), enquanto $U$. terrai possui 17 dentes (4 no pré-maxilar e 13 no maxilar).

Anatosuchus minor Sereno, Sidor, Larsson \& Gado 2003 também possui uma dentição numerosa e todos os dentes de aspecto cônico. São seis dentes pré-maxilares, dezenove dentes maxilares (ligeiramente curvados para trás e com carenas anteriores e sem serrilhas) e um dentário com vinte e um dentes (Sereno \& Larsson 2009). Além da dentição, A. minor difere de Morrinhosuchus luziae gen. nov. et sp. nov. nos seguintes aspectos: apresenta um crânio baixo e quase retangular (vista dorsal), tem um maxilar expandido lateralmente e margem lateral curva, enquanto Morrinhosuchus luziae gen. nov. et $\mathrm{sp}$. nov. a margem é retilínea (Sereno et al. 2003).

Morrinhosuchus luziae gen. nov. et sp. nov. possui um pequeno entalhe, visto lateralmente, entre o maxilar e o pré-maxilar. Tal estrutura também ocorre em Araripesuchus gomesii Price 1959, Comahuesuchus brachybuccalis Bonaparte 1991 e Malawisuchus mwakasyungutiensis; ventralmente, o pré-maxilar sobrepõe o maxilar, sem haver sutura, característica também apontada em C. brachybuccalis (Price 1959, Martinelli 2003, Gomani 1997).

Os aspectos mais marcantes de C. brachybuccalis que o difere de Morrinhosuchus luziae gen. nov. et sp. nov. são os seguintes: um dentário bastante largo e a existência de um dente hipertrofiado; rostro extremamente curto; o maxilar com uma face dorso-lateral quase horizontal, cinco dentes pequenos, exceto o terceiro hipertrofiado, e uma fossa paracanina em sua região mais posterior; um palato secundário curto e bastante largo e um contato nasal-maxilar curto, como em Malawisuchus e Simosuchus.

Simosuchus clarki apresenta inúmeras carac- terísticas únicas e distintas de Morrinhosuchus luziae gen. nov. et sp. nov., tais como: os maxilares não se tocam e consequentemente não participam da linha medial do palato. Separando estes ossos estão os vômeres e uma projeção anterior dos pterigóides. Uma extensão dorsal da pré-maxila separa as extremidades anteriores dos nasais (Buckley et al. 2000).

As espécies do gênero Araripesuchus apresentam as seguintes características que as diferem de Morrinhosuchus luziae gen. nov. et sp. nov.: dois tipos de dentes (um cônico e outro expandido antero-posteriormente com duas carenas denticuladas); o terceiro dente do maxilar é hipertrofiado, fenestra pré-orbitária de tamanho médio e um rostro ligeiramente mais largo que alto (Ortega et al. 2000).

Araripesuchus gomesii apresenta como características distintas para Morrinhosuchus luziae gen. nov. et sp. nov. uma dentição relativamente numerosa (quatro no pré-maxilar e onze dentes maxilares) uma fenestra antero-orbital grande, a maxila intumescida lateralmente na região dos grandes dentes anteriores. O esplenial tem participação de aproximadamente um terço na sínfise mandibular e o dentário é côncavo dorsalmente na região da sínfise (Price 1959).

Em Araripesuchus patagonicus Ortega, Gasparini, Buscalioni \& Calvo 2000 o esplenial corresponde a quase metade da sínfise mandibular e o dentário não é um osso muito alto. Em vista lateral observa-se um forâmen na linha de sutura maxilar e pré-maxilar. Tal forâmen poderia estar presente em Morrinhosuchus luziae gen. nov. et sp. nov., pois o maxilar e o pré-maxilar estão desarticulados e o que se se observa é um entalhe nesta região. Se fosse possível a articulação destes ossos, restaria uma pequena porção que não se articularia e resultaria em um entalhe bem mais inexpressivo ou em um pequeno forâmen.

Araripesuchus buitreraensis Pol \& Apesteguia 2005 apresenta a região rostral mais larga que alta e ligeiramente côncava dorsalmente, enquanto Morrinhosuchus luziae gen. nov. et $\mathrm{sp}$. nov. exibe um rostro mais alto que largo e em vista dorsal se mostra ligeiramente convexo (Pol \& Apesteguia 2005).

Araripesuchus wegeneri Buffetaut \& Taquet 1979 possui cinco dentes no pré-maxilar, já as demais espécies de Araripesuchus apresentam quatro dentes; o esplenial está bastante exposto ventralmente, com uma participação igual ao dentário na extensão da superfície ventral da mandíbula, característica dividida com Notosuchus e Baurusuchus (Ortega et al. 2000). Em Morrinhosuchus luziae gen. nov. et sp. nov. a exposição ventral do esplenial é bastante limitada e se mostra apenas na região da sínfise. Ocorre em $A$. wegeneri um forâmen entre o pré-maxilar e o maxilar (Sereno \& Larsson 2009), estrutura que possivelmente também ocorre em Morrinhosuchus luziae gen. nov. et sp. nov..

Araripesuchus rattoides Sereno \& Larsson 2009 possui o primeiro dente do dentário procumbente e relativamente maior que o observado em $A$. wegeneri, ambas as espécies apresentam o quarto dente do dentário hipertrofiado, embora Morrinhosuchus luziae gen. nov. 
et $\mathrm{sp}$. nov. não tenha a parte distal do dentário preservada, não há evidencias de um dente hipertrofiado no dentário. Tanto $A$. rattoides, quanto $A$. wegeneri apresentam o dentário mais largo que alto na região da sínfise mandibular; já Morrinhosuchus luziae gen. nov. et sp. nov. exibe nesta região um dentário alto e estreito.

Notosuchus terrestris Woodward 1896 exibe nasais curtos e largos. A sínfise mandibular é curta; o maxilar possui sete dentes comprimidos lábio-lingualmente, onde os quatro primeiros aumentam progressivamente de tamanho antero-posteriormente, e os demais possuem o mesmo tamanho. Há também a presença de uma fenestra maxilopalatal, diferindo de Morrinhosuchus luziae gen. nov. et sp. nov., o qual apresenta nasais longos e estreitos e uma sínfise mais longa, além de dentes sem compressão lábiolingual e ausência de fenestra maxilo-palatal.

Os notossúquios são representados por gêneros com grandes variações anatômicas, principalmente no crânio (retangulares, triangulares e de rostros bulbosos) e na dentição altamente especializada, com quase todas as espécies apresentando certo grau de heterodontia. Várias interpretações relacionadas à dieta foram elaboradas a partir da análise morfológica do crânio e sua dentição. A onivoria é proposta para a maioria das espécies, e dietas essencialmente herbívoras são propostas para algumas formas. $\mathrm{O}$ alto grau de especialização observado nas estruturas dentárias dos notossúquios indica a diversidade de hábitos alimentares, com a utilização de alimentos pouco habituais, ou mesmo inexplorados pelos crocodilomorfos até então. Tal fato permitiria a ocupação de novos nichos (Fig. 5), e possivelmente resultaria a referida especialização (Iori \& Carvalho 2008). A grande diversidade de notossúquios na Bacia Bauru pode ser um indicador da necessidade de uma busca por fontes nutricionais alternativas, desencadeada por competição ou até mesmo pela escassez de alimentos.

CONCLUSÕES A morfologia dentária de Morrinhosuchus luziae é semelhante à encontrada no gênero Mariliasuchus, no entanto a disposição e o arranjo dos dentes são distintos, e embora a distribuição de dentes pré-maxilares e maxilares em $M$. amarali não seja consensual, o espécime aqui estudado apresenta sete dentes pós-caniniforme, enquanto Mariliasuchus possui seis dentes. Além disso, várias características autapomórficas do espécime em estudo sustentam a diferença para Mariliasuchus, principalmente no arranjo geral do rostro, que se mostra bastante alto e estreito, sem nenhuma constrição, além da presença de uma fenestra antero-orbital e ausência da fenestra maxilo-palatal. A dentição e um novo arcabouço estrutural do rostro ampliam a diversidade de formas dos notossúquios gondwânicos. A definição desta nova espécie contribui assim para ampliar o conhecimento acerca dos tetrápodes e ecossistemas terrestres cretácicos da Bacia Bauru.

Agradecimentos À Antonio Celso de Arruda Campos, Sandra Aparecida Simionato Tavares (Museu de Paleontologia de Monte Alto) e Deverson da Silva (Pepi) pelo auxílio na preparação e ilustração do fóssil. Este estudo contou com o apoio do Conselho Nacional de Desenvolvimento Científico e Tecnológico (CNPq, Proc. $\left.n^{\circ} 305780 / 2006-9\right)$ e Fundação de Amparo à Pesquisa do Estado do Rio de Janeiro (FAPERJ, Proc $n^{\circ}$ E-26/102-692/2008).

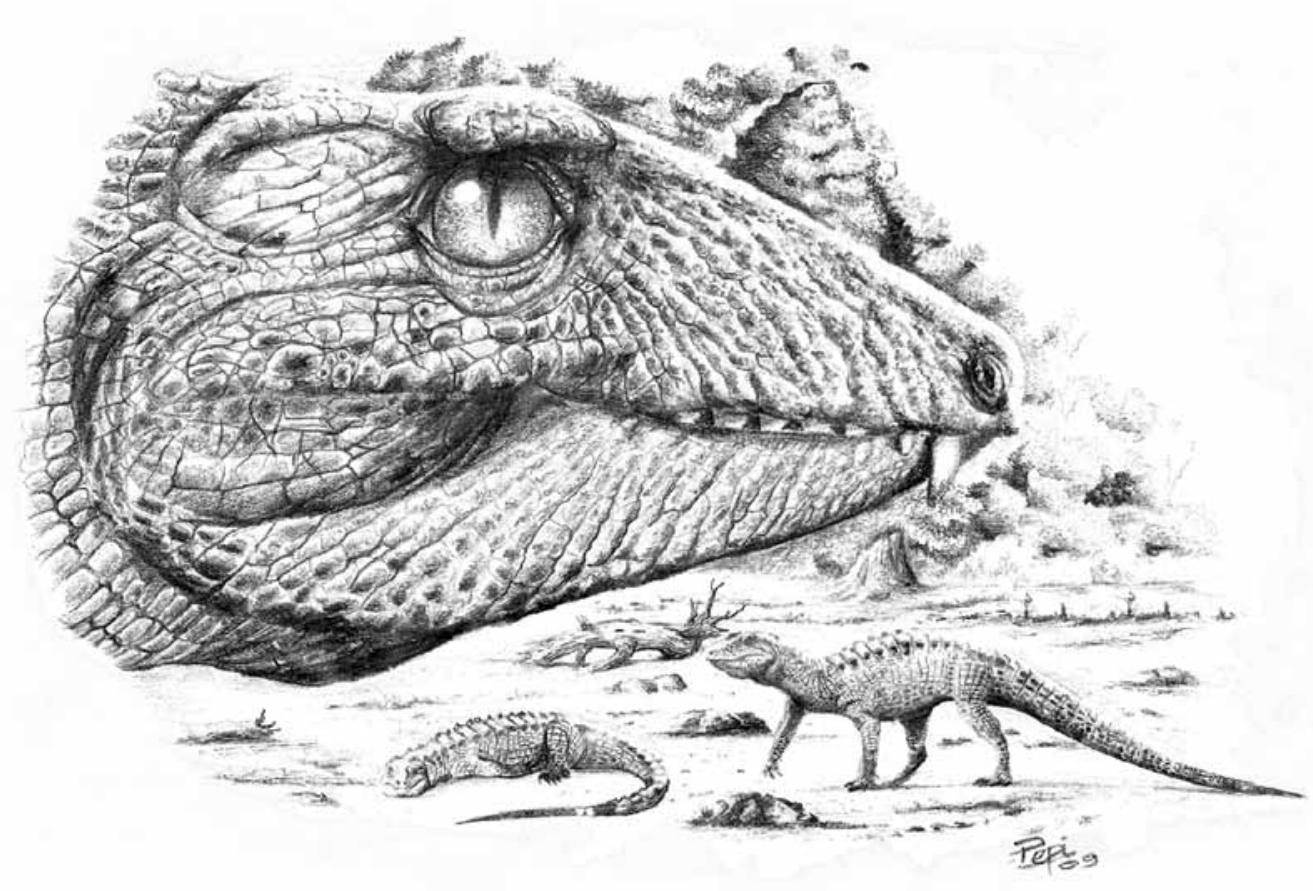

Figura 5 - Reconstrução de Morrinhosuchus luziae gen. nov. et sp. nov. em seu habitat (arte de Pepi). 


\section{Referências}

Andrade M.B. \& Bertini R.J. 2008. A new Sphagesaurus (Mesoeucrocodylia: Notosuchia) from the Upper Cretaceous of Monte Alto City (Bauru Basin, Brazil), and a revision of the Sphagesauridae. Historical Biology, 20:101-136.

Buckley G.A., Brochu C.A., Krause D.W. \& Pol D. 2000. A pug-nosed crocodyliform from the Late Cretaceous of Madagascar. Nature, 405:941-944.

Batezelli A., Saad A.R., Etchebehere M.L.C., Perinotto J.A.J. \& Fulfaro V. J. 2003. Análise estratigráfica aplica à Formação Araçatuba (Grupo Bauru - Ks) no centro-oeste do Estado de São Paulo. Geociências, 22:5-19.

Batezelli A., Gomes N.S. \& Perinotto J.A.J. 2005. Petrografia e evolução diagenética dos arenitos da porção norte e nordeste da Bacia Bauru (Cretáceo Superior). Revista Brasileira de Geociências, 35:311-322.

Carvalho I.S. 1993. Candidodon: um crocodilo com heterodontia (Notosuchia, Cretáceo Inferior). Anais da Academia Brasileira de Ciências, 66:331-346.

Carvalho I.S. \& Bertini R.J. 1999. Mariliasuchus: um novo Crocodylomorpha (Notosuchia) do Cretáceo da Bacia Bauru, Brasil. Geologia Colombiana, 24:83-105.

Carvalho I.S., Ribeiro L.C.B. \& Avilla L.S. 2004. Uberabasuchus terrificus sp. nov, a new Crocodylomorpha from the Bauru Basin (Upper Cretaceous), Brazil. Gondwana Research, 7:975-1002.

Dias-Brito D., Musacchio E.A., Castro J.C., Maranhão M.S.A.S., Suarez J. M. \& Rodrigues R. 2001. Grupo Bauru: uma unidade continental do Cretáceo no Brasil - concepções baseadas em dados micropaleonotológicos, isotópicos e estratigráficos. Rèvue Paléobiologie, 20:245-304.

Fernandes L.A. \& Coimbra A.M. 1994. O Grupo Caiuá (Ks): Revisão Estratigráfica e Contexto Deposicional. Revista Brasileira de Geociências, 24:164-176.

Fernandes L.A. \& Coimbra A. M. 1996. A Bacia Bauru (Cretáceo Superior, Brasil). Anais da Academia Brasileira de Ciências, 68:195-205.

Fernandes L.A. \& Coimbra A.M. 2000. Revisão Estratigráfica da Parte Oriental da Bacia Bauru (Neocretáceo). Revista Brasileira de Geociências, 30:717-728.

Gomani E.M. 1997. A crocodyliform from the early Cretaceous Dinosaurs Beds, Northern Malawi. Journal of Vertebrate Paleontology, 17:280-294.

Iordansky N.N. 1973. The Skull of the Crocodylia. In: Gans C. \& Parsons T.S. (eds.) Biology of the Reptilia, Londres, Academic Press, p. 201-262.

Iori F.V. 2008. Um novo Crocodylomorpha Notosuchia Sphagesauridae (Bacia Bauru, Cretáceo Superior) do município de Monte Alto, estado de São Paulo. Dissertação de Mestrado, Instituto de Geociências, Universidade Federal do Rio de Janeiro, 73p.

Iori F.V. \& Carvalho I.S. 2008. Hábitos alimentares dos crocodilyformes notossúquios: indicadores da diversidade dos ecossistemas terrestres do Cretáceo. In: SBG, Congresso Brasileiro de Geologia, 44, Anais, p.721.

Marinho T.S. \& Carvalho I.S. 2009. An armadillo-like sphagesaurid crocodylifom from the late Cretaceous of Brazil.
Journal of South American Earth Sciences, 27:36-41

Martinelli G.M. 2003. New cranial remains of the bizarre notosuchid Comahuesuchus brachybuccalis (Archosauria, Crocodyliformes) from the Late Cretaceous of Rio Negro Province (Argentina). Ameghiniana, 40:559-572.

Nobre P. H. \& Carvalho I. S. 2002. Osteologia do crânio de Candidodon itapecuruense (Crocodylomorpha, Mesoeucrocodylia) do Cretáceo do Brasil. In: Simpósio sobre o Cretáceo no Brasil, 6, Simpósio sobre el Cretácico de América del Sur, 2, Boletim, p.77-82.

Nobre P.H. \& Carvalho I.S. 2006. Adamantinasuchus navae: A new Gondwanan Crocodilomorpha (Mesoeucrocodylia) from the Late Cretaceous of Brazil. Gondwana Research, 10:370-378.

Nobre P.H., Carvalho, I.S., Vasconcellos F. M. \& Nava W. R. 2007. Mariliasuchus robustus, um novo Crocodylomorpha (Mesoeucrocodylia) da Bacia Bauru, Brasil. Anuário do Instituto de Geociências - UFRJ, 30:32-42.

Ortega F., Gasparini Z., Buscalioni A.D. \& Calvo J.O. 2000. A new species of Araripesuchus (Crocodylomorpha, Mesoeucrocodylia) from the lower Cretaceous of Patagonia (Argentina). Journal of Vertebrate Paleontology, 20:57-76.

Pol D. 2003. New remains of Sphagesaurus huenei (Crocodylomorpha: Mesoeucrocodylia) from the late Cretaceous of Brazil. Journal of Vertebrate Paleontology, 23:817-831.

Pol D. \& Apesteguia S. 2005. New Araripesuchus remains from the Early Late Cretaceous (Cenomanian-Turonian) of Patagônia. American Museum Novitates, 3490:1-38.

Price L.I. 1959. Sobre um crocodilídeo notossúquio do Cretáceo Brasileiro. DNPM/DGM, Boletim 188, p.1-55.

Rusconi C. 1933. Sobre reptiles cretaceous del Uruguay (Uruguaysuchus aznarezi, n. g. n. sp) y sus relaciones com los notosúquidos de Patagônia. Boletin Instituto de Geologia y Perforaciones Montevideo Uruguay, 19:1-64.

Sereno P.C., Sidor C. A., Larsson H.C.E. \& Gado B. 2003. A new notosuchian from the Early Cretaceous of Niger. Journal of Vertebrate Paleontology, 23:477-482.

Sereno P.C. \& Larsson H.C.E. 2009. Cretaceous Crocodyliforms from the Sahara. ZooKeys, 28:1-43.

Wu X.C. \& Sues H.D. 1996. Anatomy and phylogenetic relationships of Chimaerasuchs paradoxus, an unusual crocodyliform reptile from the Lower Cretaceous of Hubei, China. Journal of Vertebrate Paleontology, 16:688-702.

Vasconcellos F.M. \& Carvalho I. S. 2005. Estágios de desenvolvimento de Mariliasuchus amarali, Crocodyliformes Mesoeucrocodylia da Formação Adamantina, Cretáceo Superior da Bacia Bauru, Brasil. Anuário do Instituto de Geociências, 28:49-69.

Zaher H., Pol, D., Carvalho A.B., Riccomini C., Campos D. \& Nava W. 2006. Redescription of the Cranial Morphology of Mariliasuchus amarali, and Its Phylogenetic Affinities (Crocodyliformes, Notosuchia). American $\mathrm{Mu}$ seum Novitates, 3512:1-40.

Manuscrito ID 12839 Submetido em 18 de novembro de 2008 Aceito em 13 de dezembro de 2009 